\title{
Statistical observations of the MLT, latitude and size of pulsed ionospheric flows with the CUTLASS Finland radar
}

\author{
G. Provan, T. K. Yeoman \\ Department of Physics and Astronomy, University of Leicester, University Road, Leicester LE1 7RH, UK
}

Received: 15 June 1998 / Revised: 12 January 1999 / Accepted: 26 January 1999

\begin{abstract}
A study has been performed on the occurrence of pulsed ionospheric flows as detected by the CUTLASS Finland HF radar. These flows have been suggested as being created at the ionospheric footprint of newly-reconnected field lines, during episodes of magnetic flux transfer into the terrestrial magnetosphere (flux transfer events or FTEs). Two years of both hightime resolution and normal scan data from the CUTLASS Finland radar have been analysed in order to perform a statistical study of the extent and location of the pulsed ionospheric flows. We note a great similarity between the statistical pattern of the coherent radar observations of pulsed ionospheric flows and the traditional low-altitude satellite identification of the particle signature associated with the cusp/cleft region. However, the coherent scatter radar observations suggest that the merging gap is far wider than that proposed by the Newell and Meng model. The new model for cusp low-altitude particle signatures, proposed by Lockwood and Onsager and Lockwood provides a unified framework to explain the dayside precipitation regimes observed both by the low-altitude satellites and by coherent scatter radar detection.
\end{abstract}

Key words. Magnetospheric physics (magnetosphereionosphere interactions; plasma convection; solar windmagnetosphere interactions).

\section{Introduction}

The high-latitude ionospheric regions have traditionally been described in terms of the magnetospheric source regions to which they are believed to map, characterised by comparing high-altitude spacecraft plasma measure-

Correspondence to: G. Provan ments with the field-aligned plasma characteristics observed at low altitudes. Newell and Meng (1988, 1992) and Newell et al. (1989) studied data from many thousands of low-altitude polar-orbiting satellite passes, which provided meridional cuts through the highlatitude ionosphere. They derived statistically averaged mappings of the dayside ionosphere to the magnetosphere according to particle precipitation characteristics. The results are illustrated in Fig. 1 (from Newell and Meng, 1992).

Newell and Meng $(1988,1992)$ defined a 'cusp proper', a relatively limited region localised near local noon, with a typical width of 2.5 hours MLT, where the entry of magnetosheath particles was most direct. The magnetopause reconnection X-line was assumed to map to the cusp proper. They defined the region just equatorward of the cusp as the cleft/low-latitude boundary layer (LLBL) region. This region was characterised by having a slightly lower flux of particles with slightly higher energies compared to the cusp, and was believed to consist of a mixture of magnetosheath and magnetospheric particles. Newell and Meng (1988) believed that 'for many years the terms 'cusp' and 'cleft' were used interchangeably', and that this resulted in the broad cusp as identified by, among others, Heikkila and Winningham (1971).

The Newell and Meng model for mapping the dayside ionospheric regions has been criticised by Lockwood et al. (1996), Lockwood (1997) and Onsager and Lockwood (1997), for considering the ionospheric precipitation regions to be simply the field-aligned projection of the overlying magnetospheric regions. These workers considered that the Newell and Meng model neglected magnetic convection and the motion of particles in crossed electric and magnetic fields. They offered a reinterpretation of the Newell and Meng dataset, including in their new model the effect of velocity dispersion and solar wind plasma concentration on the precipitating particles.

Observations of the dayside cusp have revealed it to be a highly dynamic region of the magnetosphere. In the cusp 


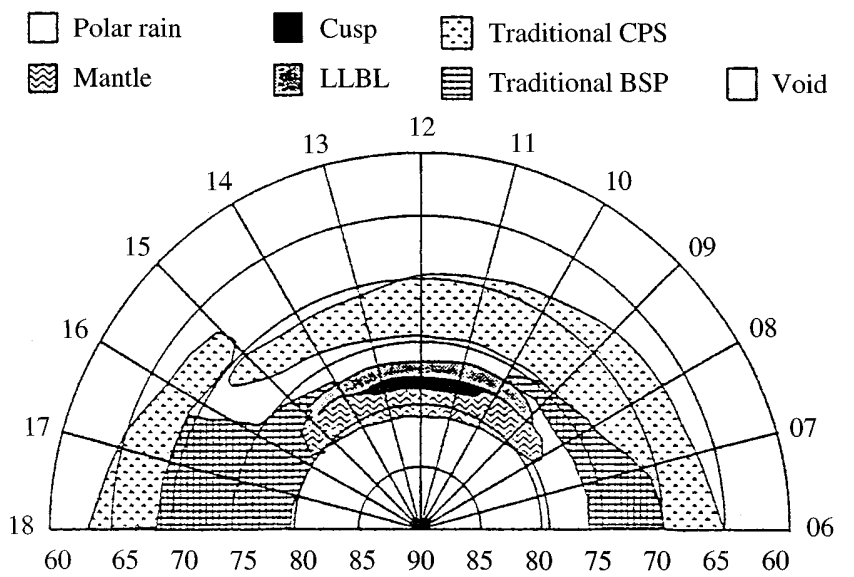

Fig. 1. A map of the high-latitude ionosphere to regions of the magnetosphere based on plasma characteristics (Newell and Meng, 1992)

region, impulsive dayside reconnection at the magnetopause boundary is believed to be the primary mechanism for the transfer of flux from the Earth's magnetosheath to the magnetosphere and episodes of such flux transfer are referred to as flux transfer events (Russell and Elphic, 1978, 1979; Elphic and Russell, 1979). Much theoretical work had been developed to explain and model FTEs at the dayside magnetopause and at the ground (Cowley, 1984; Siscoe and Huang, 1985; Cowley et al., 1991, 1992; Cowley and Lockwood, 1992; Lockwood, 1993), and Glassmeier and Stellmacher (1996) stated that 'transient reconnection at the dayside magnetopause causes an Alfvén reconnection pulse localised in space and time, which causes bursts of ionospheric flows associated with transient auroral forms and transient or wave packet-like ground magnetic variations'.

The first observation of impulsive dayside reconnection was published by Haerendel et al. (1978) using data from the HEOS-2 satellite. Russell and Elphic (1978, 1979), analysed data from the ISEE- 1 and -2 satellites and offered a detailed description of flux transfer events 'in which reconnection starts and stops in a matter of minutes or less, resulting in the ripping off of flux tubes from the magnetosphere', and offered a theoretical model for the observed magnetic signatures. The first groundbased observations of flux transfer events were published by Van Eyken et al. (1984) and Goertz et al. (1985). Goertz et al. (1985) used data from the STARE radar (a VHF coherent scatter radar sounding the $\mathrm{E}$ region ionosphere, Greenwald et al., 1978). They detected a convection boundary in the radar's field of view. Poleward of this boundary the flow was observed to be antisunward, with an occasional significant north-south component. Flows across the convection boundary occurred sporadically in spatially localised regions with scale sizes between $50-300 \mathrm{~km}$, and with a repetition rate of the order of minutes. In addition, Sandholt et al. $(1990,1992)$ observed periodic auroral events at the polar cap boundary between 0900 and 1500 MLT, seemingly the optical ionospheric signature of flux transfer events. Moen et al. (1995) used satellite data combined with optical observations and data from the EISCAT radar to observe high-latitude plasma flow variations accompanied by bursts of return flow at lower latitudes.

HF radars have been established as powerful tools for the study of the cusp. Strong HF radar backscatter with antisunward convective flows, have previously been demonstrated to be co-located with DMSP particle signatures of the cusp and poleward moving optical transients characteristic of pulsed reconnection during FTEs (Yeoman et al., 1997). Neudegg et al. (1999) observed an FTE at the magnetopause with the Equator-S spacecraft whilst corresponding transient plasma flows where observed in the near conjugate polar ionosphere by the CUTLASS Finland HF radar. Provan et al. (1998) have previously used data from the CUTLASS Finland radar to perform a detailed study of the dynamics and extent of pulsed antisunwardmoving transient features, detected poleward of a convection reversal boundary (CRB) at high latitudes in the CUTLASS field of view (f.o.v.). These pulsed ionospheric flows (PIFs) had an average recurrence rate of 7 to $8 \mathrm{~min}$. This is close to the average recurrence rate of FTEs as first reported by Rijnbeek et al. (1984). Lockwood and Wild (1993) also reported observing FTE with a mean recurrence rate of $8 \mathrm{~min}$, although the occurrence rate was highly skewed, having upper and lower decile values of $1.5 \mathrm{~min}$ and $18.5 \mathrm{~min}$ respectively. Pinnock et al. (1995) observed pulsed velocity transients in data from the Halley HF radar. The transients significantly increased the line-of-sight Doppler velocity and had an average recurrence rate of $7 \mathrm{~min}$. Rodger and Pinnock (1997) observed pulsed velocity features which were almost omni-present, but a large one occurred every 7 min. Pinnock et al. (1995) and Rodger and Pinnock (1997) identified these features as the ionospheric signatures of FTEs. The PIFs observed by the CUTLASS Finland radar were identified as the potential ionospheric signatures of FTEs, created at the footprint of newly reconnected field lines as they are pulled antisunward by the magnetosheath flow. In this work, average mappings of the ionospheric footprint of newly-reconnected field lines are derived by performing a statistical study on the occurrence of PIFs as observed by the CUTLASS Finland radar, detailing the dates, magnetic local times and latitudes over which the signatures were observed. The study incorporates data from two years of high-time resolution and normal scan data from March 1995 to February 1997.

It is important to note that when references to the cusp are made here, it is the narrow definition of the 'cusp proper' as proposed by Newell and Meng (1988) which is being described. The data presented in this work suggest that the cusp proper is only a subset of the region of newly reconnected field lines.

\section{Observations}

\subsection{Selection of radar data}

The ionospheric convection velocities in this study are provided by the CUTLASS Finland radar. CUTLASS is 
a bistatic HF coherent radar, with stations in Finland and Iceland, and forms part of the international SuperDARN chain of HF radars (Greenwald et al., 1995). Each radar of the system is a frequency agile (8$20 \mathrm{MHz}$ ) radar, routinely measuring the line-of-sight (1o-s) Doppler velocity and spectral width of, and the backscattered power from, ionospheric plasma irregularities. The radars each form 16 beams of azimuthal separation $3.24^{\circ}$. Each beam is gated into 75 range bins, each of length $45 \mathrm{~km}$ in standard operations, when the dwell time for each beam is $7 \mathrm{~s}$, giving a full 16 beam scan, covering $52^{\circ}$ in azimuth and over $3000 \mathrm{~km}$ in range (an area of over $4 \times 10^{6} \mathrm{~km}^{2}$ ), every $2 \mathrm{~min}$. Commonvolume data from the two stations can be combined to provide convection velocities perpendicular to the magnetic field, although no common volume backscatter was used during the intervals under study here.

Provan et al. (1998) have previously observed PIFs in one day of high-time resolution (14 s) data from the CUTLASS Finland radar. The PIFs appeared as pulsed antisunward moving transient features observed poleward of a CRB in the radar's field of view, with westward antisunward flow at higher ranges and eastward sunward flow lower ranges. A beam-swinging technique was used to measure the plasma velocities poleward of the CRB. The observed velocities supported the view that the PIFs were the potential ionospheric signatures of FTEs, created at the footprint of newly reconnected field lines as they are pulled antisunward by the magnetosheath flow.

Equatorward of the CRB continuous backscatter was observed with the magnitude of the 1-o-s velocity having a quasi-periodic nature. By measuring the spectral width poleward and equatorward of the CRB and comparing this with HF radar signatures of the LLBL and the cusp as defined by Baker et al. (1995), the authors were able to identify the polar cap boundary in the pre-noon sector in the CUTLASS f-o-v, with antisunward flow on open field lines within the polar cap and sunward flows on lower latitudes on closed field lines. The quasiperiodic nature of the magnitude 1-o-s velocity equatorward of the CRB is probably created due to the modulation of the return flow on closed field lines over a time period of less than a minute (see Pinnock et al., 1995).

The same techniques used by Provan et al. (1998) have been employed on the statistical dataset presented here to identify the CRB and PIFs poleward of the CRB. The results do not prove that the CRB is necessarily collocated with the open/closed field line boundary, the position of this boundary in the cusp region is an active area of research where no firm consensus has been reached. Recent work by Lockwood (1997) has place the open/closed boundary equatorward of the CRB.

The initial part of this survey involved a study of the latitude-time-velocity (LTV) plots for two years of CUTLASS Finland high-time resolution data. The initial part of this survey involved a study of the CUTLASS Finland high time resolution (HTR) data obtained over the interval March 1995 to February 1997. HTR modes of the radar camp on between 1 and 3 beams instead of the entire sixteen, for example the radar could scan beams $5,12,5,12,5,12, \ldots$ or beams $0,5,1,5,2,5, \ldots$. With a dwell time of $7 \mathrm{~s}$ for each beam, this results in a time resolution of between 7 and $21 \mathrm{~s}$ for the HTR beams. HTR scans are run irregularly for up to $30 \%$ of each month. During the above two year interval, there were 113 days of HTR data. Latitude-timevelocity plots of the 1-o-s velocity were studied for these days. A total of 31 days were judged to very clearly display PIF signatures, appearing as pulsed antisunward-moving rapid velocity transients with a typical recurrence rate of 7-8 $\mathrm{min}$ (varying between 5 and $12 \mathrm{~min}$ ). The absolute minimum value of the l-o-s velocity of the PIFs was $400 \mathrm{~ms}^{-1}$ (directed either away from or towards the radar), if background flow was present the PIFs increased the 1-o-s velocity by at least $200 \mathrm{~ms}^{-1}$ over a time period of less than a minute (see Pinnock et al., 1995).

Figure 2 illustrates the LTV plot for beam 5 for one of these 31 days, for the interval $0530-0900$ UT on 4 August, 1996. For beam 5 at these latitudes $\mathrm{MLT} \approx \mathrm{UT}+2$. The transients appear as pulsed red stripes and are observed poleward of a convection reversal boundary (CRB), where the l-o-s velocity changes from positive values (green and blue, towards the radar) to negative values (yellow and red, away from the radar), some of the transients are marked with black lines. The transients are observed from $\sim 0610$ to 0830 UT ( 0810-1030 MLT) between the magnetic latitude $75^{\circ}$ to $81.5^{\circ}$ (where we use altitude-adjusted corrected geomagnetic, AACGM, co-ordinates, based on the PACE geomagnetic co-ordinates of Baker and Wing, 1989). The direction of plasma flow either side of the CRB is consistent with the radar observing a typical southward IMF dawn cell convection, with the plasma rotating in an anti-clockwise direction.

For the 31 days of high-time resolution data, the occurrence distribution of pulsed transients features as a function of magnetic latitude and magnetic local time was calculated. Figure 3 presents the occurrence distribution of PIFs observed with high-time resolution data. The PIFs were mainly observed between 0800 and 1210 MLT, in a region stretching from $76^{\circ}$ to $82^{\circ}$ magnetic latitude. PIFs can, however, be observed during the entire interval between 0250 and 1650 MLT, between magnetic latitudes of $74^{\circ}$ and $88^{\circ}$. This demonstrates that although it is most likely that PIFs will be observed in a region in the pre-noon sector, they can actually be observed over $14 \mathrm{~h}$ of local time in a region covering $12^{\circ}$ of magnetic latitude.

As there were only 31 days of high-time resolution data in total and no data at all for certain months, it became a concern that the data sample was not large enough to offer a statistically significant result. It was also impossible to do a study of e.g. occurrence rate of PIFs per month. The possibility of using normal-scan ( $2 \mathrm{~min}$ resolution) data to identify the PIFs was therefore considered. Normal-scan data have a resolution of 2 min for each beam, which is a borderline resolution for detecting features which last for only $8 \mathrm{~min}$. A study was performed where high-time resolution data depicting 
CUTLASS FINLAND - 4 AUGUST 1996

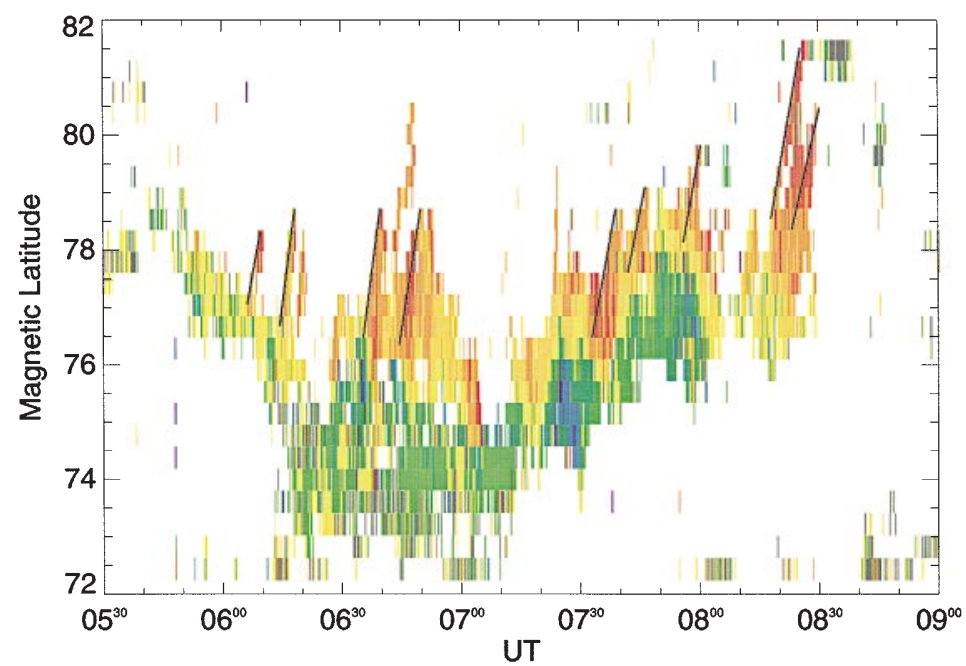

Beam 5

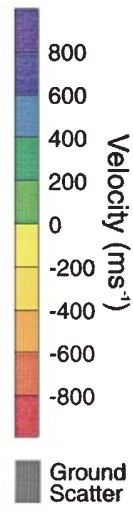

Fig. 2. Latitude-time-velocity (LTV) plots for beam 5 of the CUTLASS Finland radar, between 0530 and 0900 UT on the 4 August 1996. The Doppler velocity is colour-coded with positive velocities indicating motion towards the radar and negative velocities away from the radar. Overplotted black lines trace the motion of some of the individual transients several pulsed transients, was transformed into a simulation of normal scan data by removing seven out of every eight data points. A comparison of the two datasets demonstrated that the transient signatures are still observable in the latitude-time-velocity plot of normal resolution. Although the resolution of a normal scan is too coarse to allow a study of the dynamics of the pulses as performed by Provan et al. (1998), normal scans can nevertheless be used to investigate their occurrence.

A study was undertaken of two years of data, presented in the form of LTV plots, for beam 5 of the Finland radar when the radar was run in a normal scan mode. The two year period was the same interval previously studied with high-time resolution data. As before, only days during which PIFs could be observed very clearly were included in the study. On some days it was difficult to determine whether the observed flow was due to pulsing transients poleward of the CRB or the pulsating return flow on the equatorward side of the boundary. On these days radar data from two beams were studied instead of just one, one of the beams pointing in an eastward direction and the other beam pointing in a westward direction. On many occasions, observing the flow patterns from two different directions helped determine the position of the CRB and thus resolve any ambiguity in the observations.

\subsection{Statistical characteristics of PIFs}

During the 2 years, there were 376 days of normal scan data, a total of 132 days clearly displayed PIF signa-

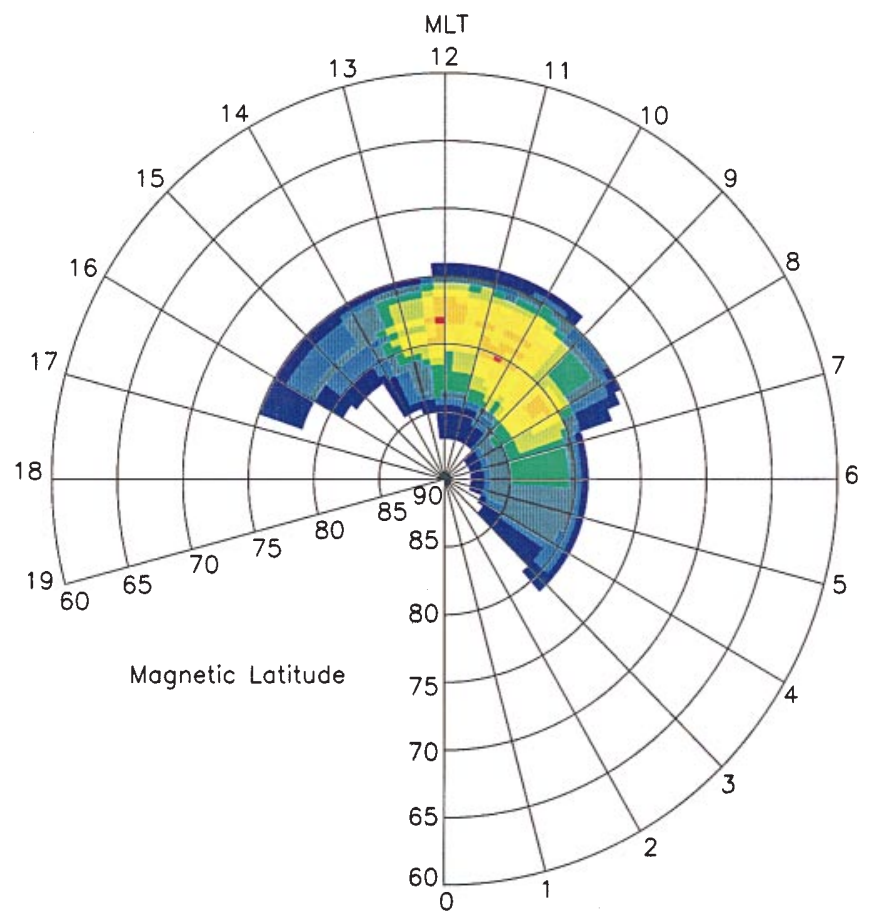

Number of Observations

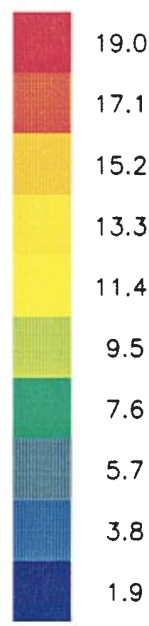

Fig. 3. Occurrence distribution in latitude and MLT of pulsed ionospheric flow (PIF) signatures, as observed by the CUTLASS Finland radar from March 1995 to February 1997 when run in high-time resolution mode 
tures. The occurrence distribution of PIFs as a function of magnetic latitude and MLT was calculated for these 132 days. The occurrence distribution for normal resolution data was very similar to the occurrence distribution obtained with high-time resolution data. Figure 4 presents the combined occurrence distribution of the ionospheric signatures of pulsed transients for both the normal scan and high time resolution data. The distribution illustrates that the PIFs can be observed for approximately $12 \mathrm{~h}$ of local time centered in the prenoon sector and over approximately $10-15^{\circ}$ of magnetic latitude, but that the signatures are most likely to be observed over a latitudinally narrow region extending over a number of hours of MLT in the pre-noon sector. The occurrence plots in Figs. 3 and 4 are analysed quantitatively below.

The effect of the IMF $B_{y}$ component on this average distribution is discussed in Provan et al. (1998). They showed that the statistical location of PIFs is strongly dependent on IMF conditions, moving to the pre-noon sector during intervals of positive IMF $B_{y}$ and into the post-noon sector during intervals of negative IMF $B_{y}$. The data in Fig. 4 are recorded predominantly under positive IMF $B_{y}$ conditions, and hence are shifted dawnwards of noon. The PIFs can be observed for approximately $11-12 \mathrm{~h}$ of local time and over $10-15^{\circ}$ of magnetic latitude during intervals of both positive and negative $B_{y}$.

As stated, there were 132 days of normal resolution data which clearly displayed PIF signatures, for each of these days we measured the magnetic latitudinal extent over which the transient signature could be observed by beam 5 of the radar. From the magnetic latitudinal extent of the individual signatures, it was possible to determine the MLT extent of the PIF signatures.
Figure 5a illustrates the distribution of the magnetic latitudinal extent of the PIF signatures for these days. The peak of the distribution is between $3^{\circ}$ and $4^{\circ}$ magnetic latitude, $70 \%$ of the PIFs have a latitudinal extent of between $3^{\circ}$ and $7^{\circ}$ magnetic latitude. Figure $5 \mathrm{~b}$ displays the distribution of the MLT extent of the PIF signatures. The distribution has a peak at 2.5-3 hours.

The two years of normal scan data were used to investigate whether there were any seasonal variations in the occurrence of PIFs. Figure 6 illustrates the normalised occurrence per calendar month on which PIFs were observed with normal scan data (black line with overplotted triangles). PIFs are observed most frequently around the equinoxes (September and March/April) and less frequently during the solstices. Figure 7 illustrates the average magnetic local time of the initial observation of the poleward moving transients as a function of calendar month. The transient signatures are initially observed earliest in the day during early summer (0700 MLT in April) and latest during mid-winter (1130-1200 MLT in December/January).

In order to determine the total duration of magnetic local time during which an individual sequence of PIF signatures could be observed by the CUTLASS Finland radar, data recorded by multiple radar beams were utilised. For each day of normal scan data during which transient signatures were observed in beam 5, LTV plots were produced for a number of beams. From these LTV plots an estimate of the UT of the initial observation of the first PIF signature (start time) and final observation of the last signature (end time) was determined. Polar plots depicting the backscatter velocity observed by all the radar beams at any one instant of time, overlaid on an axis showing geomagnetic latitude and magnetic local time, were then plotted in order to accurately derive the

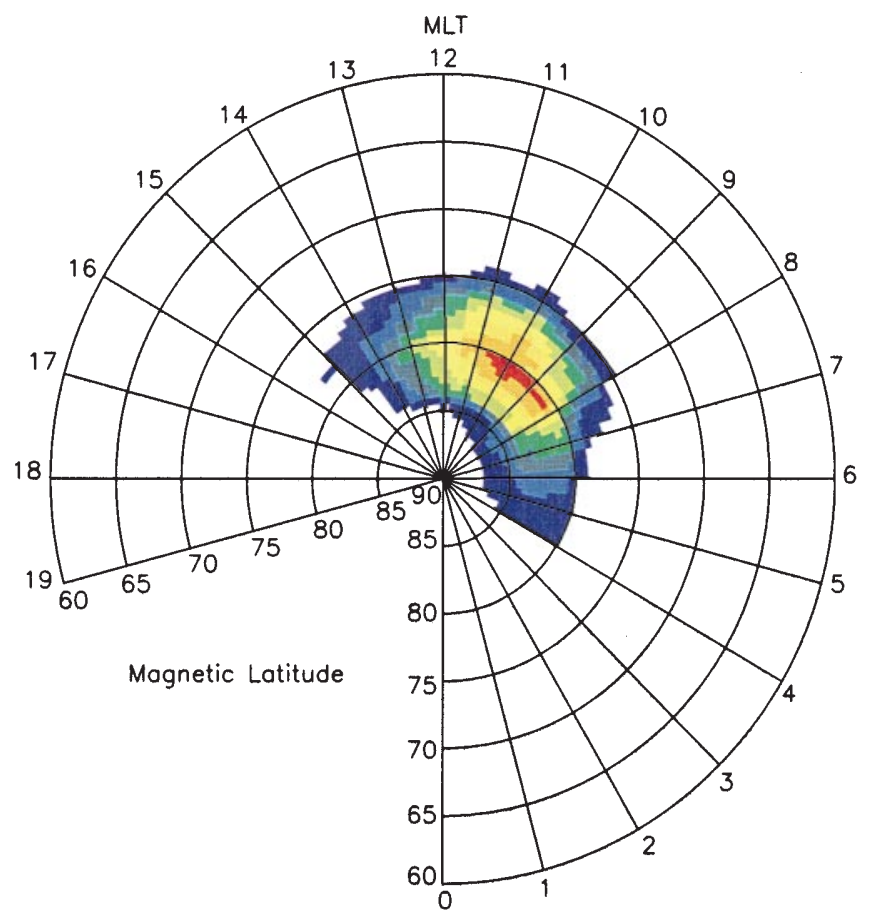

Number of Observations

95.0

85.5

76.0

66.5

57.0

47.5

38.0

28.5

19.0

9.5
Fig. 4. Occurrence distribution in latitude and MLT of PIF signatures, as observed by the CUTLASS Finland radar from March 1995 to February 1997 when run in high-time and normal resolution mode 

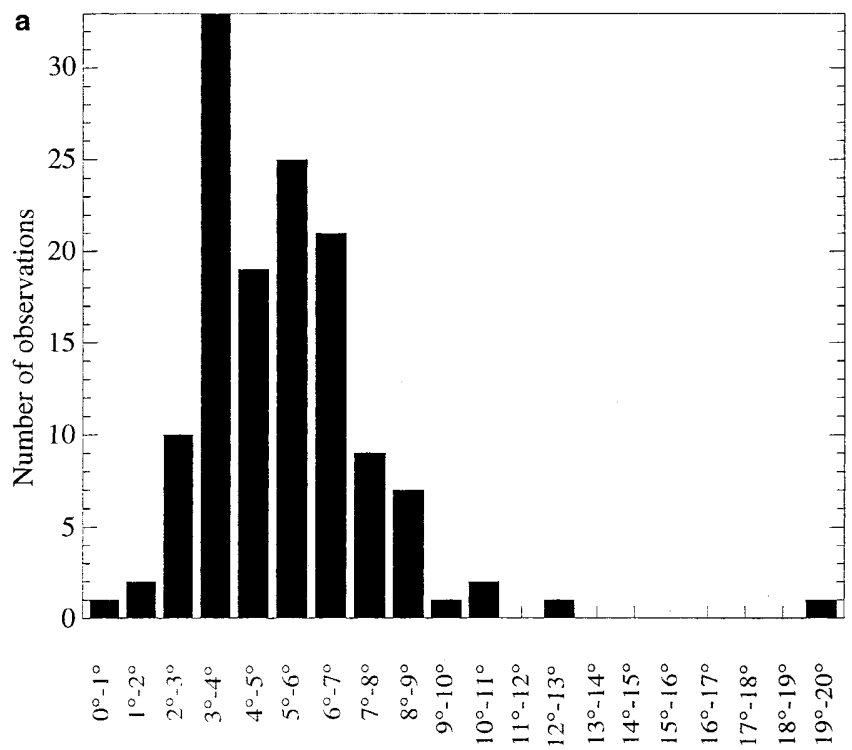

Latitudinal extent (AAGCM co-ordinate system)

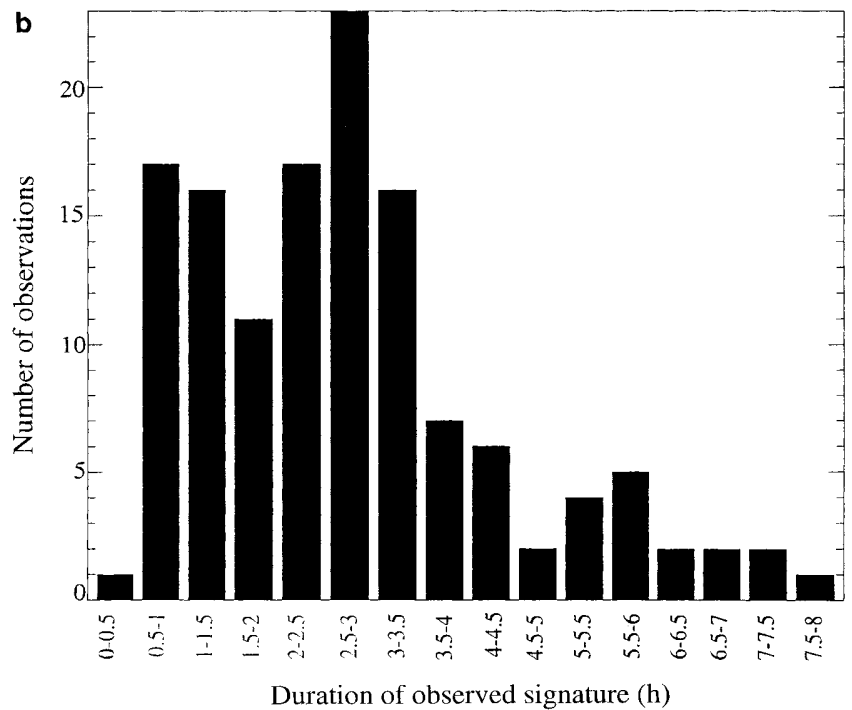

Fig. 5a, b. Distribution of a the magnetic latitudinal extent and b MLT duration of PIF signatures observed by beam 5 of the CUTLASS Finland radar, for the subset of events observed when the radar was run in a normal scan mode

start and end magnetic local times of the PIF signatures. Usually, a number of polar plots produced for different times were studied before finding the two plots from which the start and end times of the signatures could be inferred most accurately.

From the 132 days of normal scan data for which PIF signatures were detected with beam 5, it was possible to use polar plots to estimate the MLT duration of a sequence of PIF signatures for 98 separate days. As an example, Fig. 8 depicts four LTV plots and two polar plots for data recorded on the 6 March, 1995, one of the 98 days included in the study. The LTV plot depicts backscatter velocity recorded on beams 2, 6, 11 and 12 between 0500 and 1100 UT. The LTV plots demonstrate that the sequence of PIF signatures are initially observed in beam 2 at 0640 UT and finally observed in beam 11 at

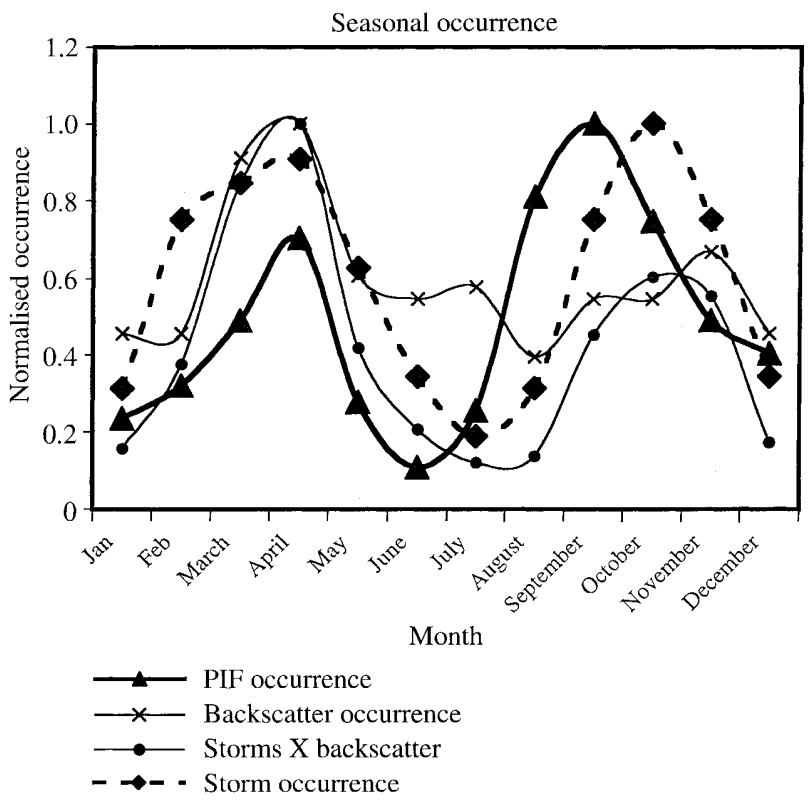

Fig. 6. Normalised occurrence of days per calendar month from March 1995 to February 1997 on which PIF signatures were observed by the CUTLASS Finland radar when run in a normal scan mode, compared with the occurrence of backscatter from the CUTLASS Finland radar and the occurrence rate of geomagnetic storms

0848 UT, these times are marked with vertical dashed lines on the LTV plots. Two polar plots for 0640 UT and 0848 UT are also shown. Beam 2 (marked with a black line on the polar plot produced at 0640 UT) detected the PIF signature first at $\sim 0700$ MLT, while beam 11 (marked with a black line at the polar plot produced at $0848 \mathrm{UT}$ ) observed the final PIF signature at $\sim 1300$ MLT. So for this day PIF signatures were observed for a duration of $6 \mathrm{~h}$ of MLT.

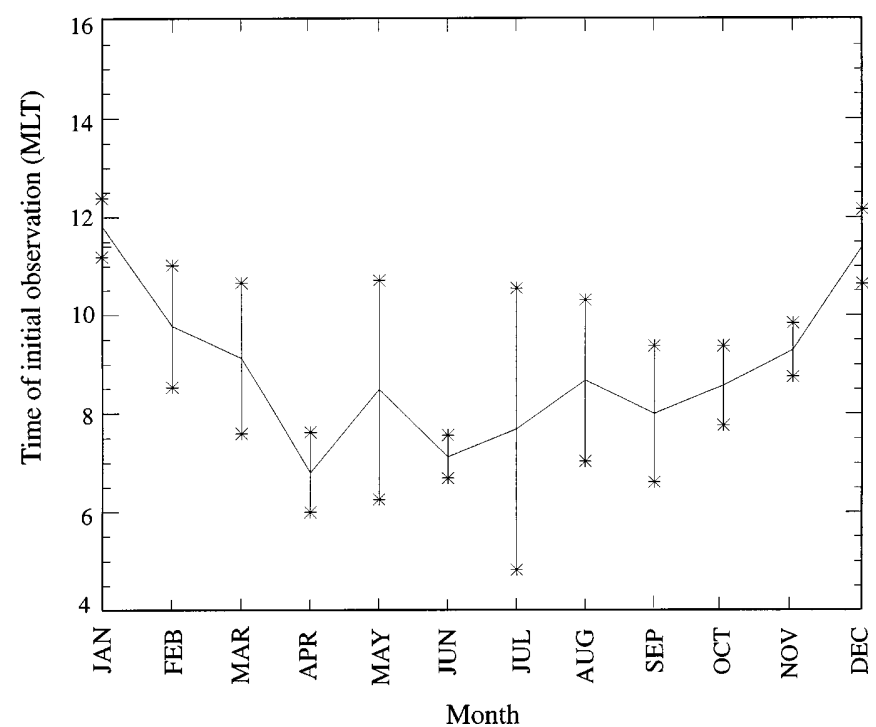

Fig. 7. Average time of initial observation of PIF signatures, for the subset of events observed by the CUTLASS Finland radar when run in a normal scan mode. The observations were made between March 1995 and February 1997 
The azimuthal extent of the sequence of PIF signatures was calculated for the 98 days of normal scan data. The estimated average latitude at which the transients were observed was $77^{\circ}$ (one hour of MLT at a latitude of $77^{\circ}$ is equal to $375 \mathrm{~km}$ in an azimuthal direction). The duration of the transient in MLT has been converted into the extent of the transient signature in the azimuthal direction. Figure 9 presents the distribution of the azimuthal extent of the sequence of PIFs in MLT and $\mathrm{km}$, as inferred from polar plots for 98 days of data. The transient signatures can be observed for a duration of between $0-0.5$ and $12.5-13 \mathrm{~h}$. The peak of the distribution is between 4 and $4.5 \mathrm{~h}$, whilst for $28 \%$ of observations the ionospheric signature of PIFs can be observed for a duration of between 8 and $13 \mathrm{~h}$. In the case of the remaining 34 days for which the azimuthal extent of the signature could not be estimated, this was either because the radar, although scanning in the normal scan mode on beam 5, was not scanning on all the beams in this way, or simply because the start and end times of the transient signatures could not be clearly identified from the polar plots.

\section{Discussion}

In the introductory section we discussed the concept of using PIF signature detected by the CUTLASS Finland HF radar (hereto said to be the ionospheric signatures of FTEs) as a means of identifying the ionospheric signatures of newly reconnected field lines. The PIF signatures do not necessarily map to the 'cusp proper', rather they give an estimate over the extent that newly reconnected field lines convect while still being detected by the HF radar. Figure 4 demonstrates the probability of the observing the signature of newly reconnected field lines with high time and normal resolution radar data. The plot illustrates that although the signature of newly opened flux is most likely to be observed in the pre-noon sector in a latitudinally-narrow region stretching for a few degrees, newly open field lines can be identified over approximately $12 \mathrm{~h}$ of local time, and over a wide latitudinal range.

Using data from all the beams of the CUTLASS radar offers the possibility to observe the ionospheric signature of newly reconnected field lines simultaneously for up to $8 \mathrm{~h}$ of local time. Figure 9 illustrates the distribution of the azimuthal extent in MLT and equivalent kilometres of the ionospheric footprint of the newly reconnected field lines. In this case the newly reconnected field lines were observed only with normalresolution data. The distribution had a peak at $4-4.5 \mathrm{~h}$ in magnetic local time $(\sim 1500 \mathrm{~km})$, while for $28 \%$ of observations the PIFs extended between 8 and $13 \mathrm{~h}$ magnetic local time (3000-4700 km). These observations offer a minimum extent of ionospheric footprint of newly reconnected flux, the dataset being constrained by the limited field-of-view of the radar, and the sporadic nature of the FTE process itself.

There are, in fact, a number of potential sources of errors that have to be considered when mapping the ionospheric footprint of newly-reconnected field lines using this technique. Firstly, a finite value of IMF $B_{y}$ can lead to the size of the region of newly reconnected field lines being over-estimated. Secondly the size of the region of newly reconnected field lines may be over- or underestimated due to the temporal and spatial variability of the region of open flux, for example due to the changing IMF (on average every $20-40$ minutes) or the rotation of the region of newly reconnected field lines with the sun, this will be investigated further in Sect. 3.1. Thirdly, it is possible that the backscatter may be created by precipitation from source regions other than the cusp (on mantle field lines), resulting in an overestimation of the extent of the ionospheric footprint of the newly reconnected field lines.

Fourthly, the ionospheric signatures of open field lines are created due to the enhanced ionisation of the ionosphere by the precipitation of particles associated with newly opened field lines. As the ionisation may persist after the precipitation has ceased, and will be convecting away from the source region, this may lead to the extent of the ionospheric footprint of newly reconnected field lines being either overestimated or underestimated, depending on the geometry of the radar beams and the orientation of the $B_{y}$ component of the IMF.

We have attempted to predict the significance of these errors when using FTE signatures to map the ionospheric footprint of newly reconnected field lines. A finite IMF $\mathrm{B}_{y}$ component moves the PIF azimuthally as it moves poleward, leading to an overestimate in MLT of the region of newly reconnected field lines. Using the empirical electric field model presented by Heppner and Maynard (1987), we have estimated that for a transient initially at $75^{\circ}$ magnetic latitude at $10 \mathrm{MLT}$, observed under positive IMF $B_{y}$ conditions in the Northern Hemisphere $(3+\leq K p \leq 4-)$, the transient may have moved up to $2 \mathrm{~h}$ in MLT by the time it reaches $80^{\circ}$ magnetic latitude. Provan et al. (1998) have previously presented a comparison of the velocity of the region of radar backscatter forming the PIFs with the measured plasma convection velocity. These two velocities were not equal, suggesting that the patch of newly reconnected field lines map to still active reconnection regions. In addition the study of Yeoman et al. (1997), demonstrated that there is an excellent degree of colocation between radar backscatter, DMSP and optical cusp signatures. Rodger et al. (1995) used simultaneous optical all-sky imager and photometer data together with $\mathrm{HF}$ radar data to prove that their respective ionospheric signatures of the magnetospheric cusp are collocated to better than $1^{\circ}$ latitude. Observing the ionospheric footprint of the newly reconnected field lines simultaneously over a wide range of magnetic local time, as done in this study, would appear to be the optimum way of reducing the uncertainties involved in estimating the spatial extent of the region of newly reconnected flux.

\subsection{Comparison with previous observations}

The results presented in this study illustrate that although the signature of newly opened flux is most 

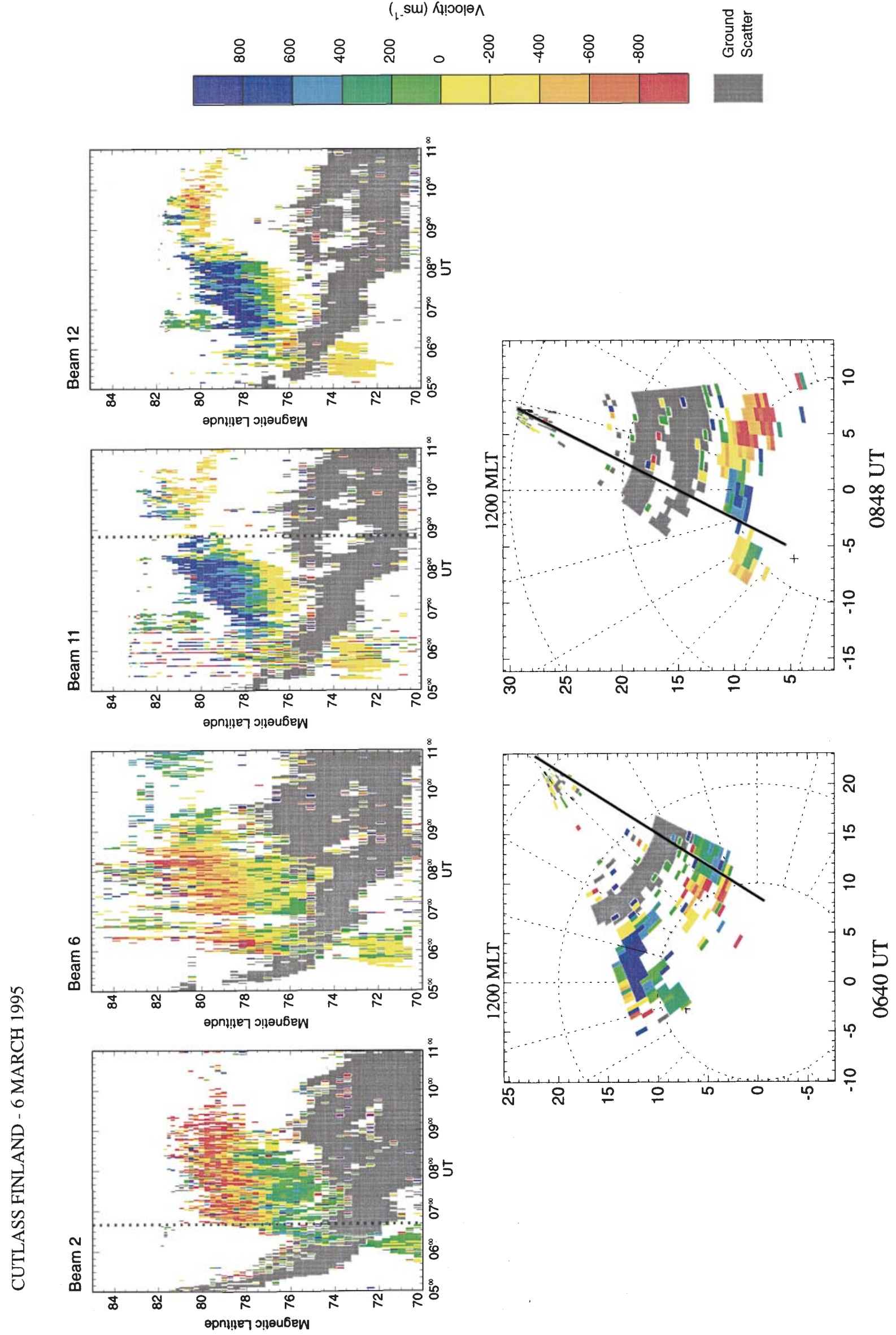
Fig. 8. a Latitude-time-velocity plots for beams 2, 6, 11 and 12 for the CUTLASS Finland radar, recorded on the 6 March 1995, 0500 to 1100 UT. A number of pulsed transients can be observed in each beam. The dashed vertical lines in beams 2 and 10 mark the first and final observation of the transient signatures with respect to MLT as determined from the plots. b Polar plots depicting the backscattered velocity observed at any one instance of time over a full radar scan, overlaid on an axis showing geomagnetic latitude and magnetic local time for 1-o-s velocity observed on 6 March 1995, at 0640 and 0848 UT. Beam 2 and beam 10 are marked with black lines in the polar plots. The transient signature can be observed for $6 \mathrm{~h}$ of MLT

likely to be observed in the pre-noon sector in a latitudinally narrow region stretching for a few degrees, newly open field lines can be identified over approximately $12 \mathrm{~h}$ of local time and over a wide latitudinal range. The PIFs observed by the CUTLASS Finland radar have previously been identified as the potential ionospheric signature of FTEs (Provan et al., 1998, 1999). Previous work by Russell et al. (1996), has observed FTEs over a wide range of MLT. By studying the occurrence rate of FTEs in the magnetosphere and the magnetosheath, they discovered that for southward IMF, FTEs occurred almost uniformly between 0500 and 1900 UT. Provan et al. (1999) have previously also observed a very wide distribution shifted toward dawn during intervals of positive IMF $B_{y}$.

As described earlier, Newell and co-workers identified the typical duration of the cusp to be about $2.5 \mathrm{~h}$ in total local time extent, an LLBL which typically extended from about 0900 MLT to 1500 MLT, and a mantle which stretches from 0800 to 1600 MLT. When

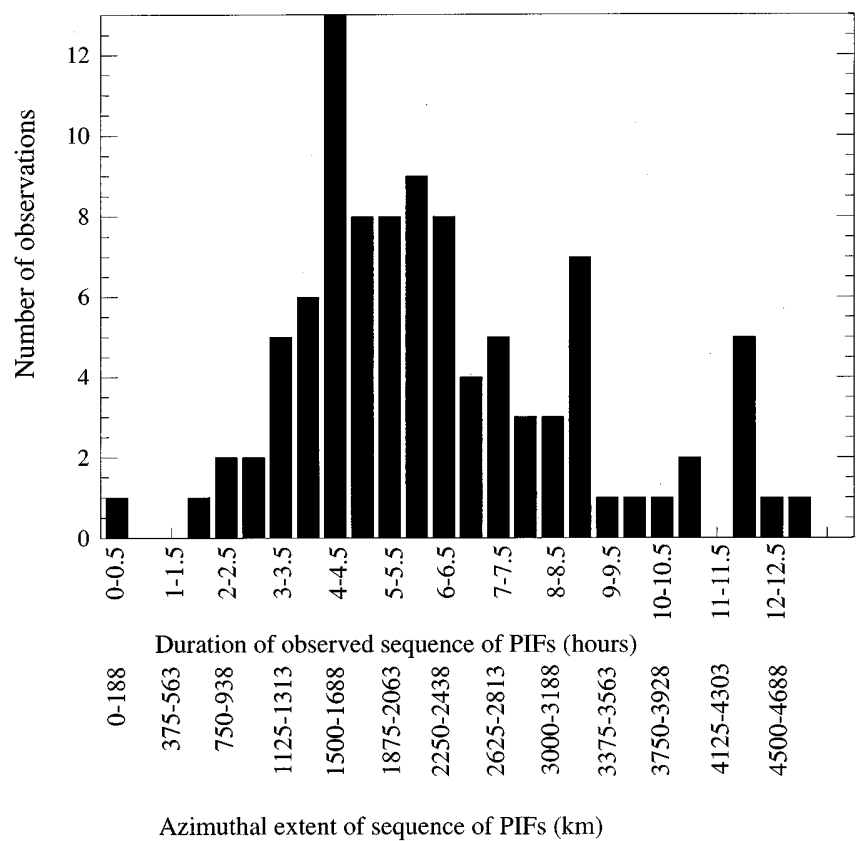

Fig. 9. Azimuthal extent of the sequence of PIFs in MLT and the equivalent size in $\mathrm{km}$, calculated from observations made using the entire f.o.v of the CUTLASS Finland radar when run in a normal scan mode (see text for details) comparing the particle distributions calculated by Newell and Meng (1992) with the occurrence distribution of PIFs derived from both the normal and high-time resolution radar data (as presented in Fig. 4), the similarity of the spatial and temporal extent of the two distributions is striking. The radar and satellite data both appear to observe enhanced precipitation for approximately $12 \mathrm{~h}$ of local time and $15^{\circ}$ of magnetic latitude. The peak in the occurrence distribution in Fig. 4, suggests that the ionospheric signature of open field lines as observed by HF radar is most likely to be observed over a number of hours of local time in a latitudinally narrow region, an area very similar in extent and duration to the region identified as the "cusp proper' by Newell and Meng (1988, 1992). Areas surrounding the 'cusp proper' have been identified as the cusp/cleft region by Newell and Meng (1992). However the radar data presented here suggests that the ionospheric signatures of newly opened field lines are often observed in these regions, just less frequently than at the pre-noon peak.

From low-altitude satellite data Newell and Meng (1992) believed a typical cusp to be observable for $2.5 \mathrm{~h}$. However the satellite results represent a typical location of the cusp, found by studying over 60000 satellite passes which recorded observations every $100 \mathrm{~min}$, and therefore does not directly signify the temporal extent of the cusp at any given moment. As stated in Sect. 3, it is not claimed that the observations presented here directly represent the temporal extent of the region of newly reconnected field lines, although we do believe that the large f.o.v of the CUTLASS radar offers considerable advantages when determining the size of the ionospheric footprint of the newly reconnected field lines, as can be illustrated by comparing Figs. $5 \mathrm{~b}$ and 9 .

Figure $5 \mathrm{~b}$ depicts the occurrence distribution of the magnetic latitudinal extent of the PIFs observed by only beam 5 of the radar when run in a normal scan mode. The distribution has a peak at 2.5-3 h, similar to the extent of the 'cusp proper' as identified by Newell and Meng (1988, 1992), but $2 \mathrm{~h}$ less than the peak in the distribution of the duration of PIFs derived using the whole field of view of the radar (see Fig. 9). These results would suggest that single-point measurements can result in the extent of the magnetospheric source regions of newly reconnected field lines being underestimated, probably due to the temporal variability of the high-latitude ionosphere. Thus, it is probable that the spatial extent of the ionospheric footprint of newly reconnected flux is greater than the size of the cusp proper as predicted by Newell and Meng $(1988,1992)$.

\subsection{Comparison with theoretical models}

Newell and Meng (1992) viewed the low-altitude particle population to be the field aligned projection of the particle populations of the overlying magnetospheric regions, and thus used them to attempt a classification and identification of the dayside ionosphere. The basic regimes have been defined as central plasma sheet (CPS), 
boundary plasma sheet (BPS), low latitude boundary layer (LLBL), cusp, mantle and polar rain, with the CPS traditionally lying on closed field lines, and the cusp, mantle and polar rain on open field lines. Debate remains over the nature of the BPS and LLBL field lines.

Lockwood et al. (1996), Lockwood (1997) and Onsager and Lockwood (1997) criticised this model of dayside particle precipitation for failing to take into account magnetospheric convection and the time-offlight of magnetospheric ions when considering particle precipitation in the low-altitude ionosphere. Onsager and Lockwood (1997) stated that as a result of the velocity filter effect "not only does a spectrum of different energy particles (at one pitch angle) from one point in the magnetosphere map to a spread of locations in the ionosphere, but also a spread of energies seen at any one point in the ionosphere maps to a spread of source locations in the magnetosphere'.

Lockwood (1997) and Onsager and Lockwood (1997), offered reinterpretation of the particle classifications produced by Newell and Meng (1992), involving a detailed description of the consequences of the velocity filter effect on the observed precipitation regions. The most energetic ions and electrons predicted from the model, observed first after reconnection, would be classified as CPS in the scheme of Newell and Meng (1992). This modelling then predicts that the CPS, traditionally seen as lying on closed field lines, is in fact partially on open field lines. Slightly less energetic precipitation, which would have been classified as BPS or LLBL in the traditional system, are predicted by the authors to lie well into the open field line region.

Newell and Meng (1992) determined the extent of the cusp proper by a threshold flux count of low energy ions and assumed that the ionospheric projection of the merging gap (defined as the cusp) was proportional only to its length on the magnetopause. Crooker et al. (1991) modelled the length of the ionospheric projection of the merging gap. They concluded that for a given merging line length at the magnetopause, the longitudinal extent of its projection in the ionosphere would vary with the merging rate. The cusp would span the dayside of the polar cap boundary from $1 \mathrm{~h}$ either side of local noon for a polar cap potential of $20 \mathrm{kV}$, to $3 \mathrm{~h}$ either side of local noon for a cross-polar cap potential of $170 \mathrm{kV}$, a value representative of strongly southward IMF e.g. Reiff et al. (1985). This value is consistent with the width of the region of poleward flow across the CRB in convection models such as Heppner and Maynard (1987). Crooker et al. (1991) stated that the predicted local time span of the cusp seems broad compared to the commonly held view that cusp particles precipitated in a localised region around local noon as proposed by Vasylinuas (1979)'. They believed that the under-estimation of the size of the cusp was due to previous workers assuming that the cusp was a field-aligned projection of the active $\mathrm{X}$-line.

Lockwood (1997) stated that, as the particle flux density in the ionosphere reflects the particle flux density in the source magnetosheath plasma connected to the linking field lines, then the apparent extent of the cusp as measured using the Newell and Meng technique will vary with solar wind concentration in both the latitudinal and longitudinal directions. Thus, the traditional technique for the identification of the extent of the cusp proper is, to some extent, arbitrary. Lockwood (1997) also modelled the poleward flow speeds, which would be initiated by applying transpolar voltages across the active X-line. A typical transpolar voltage $(100 \mathrm{kV}$ for a southward IMF) applied to a cusp whose longitudinal width is described by Newell and Meng (1992), would support a typical poleward flow speed of $3 \mathrm{kms}^{-1}$. This is approximately 5 or 6 times larger than the typical poleward flow speeds already observed (Pinnock et al., 1995; Provan et al., 1998). Lockwood (1997) suggested that the reconnection merging gap might in fact be five or six times larger than the cusp proper as identified by Newell and Meng (1992).

There is great similarity between the statistical pattern of the occurrence of pulsed antisunward moving backscatter regions which are identified here as the coherent radar signature of newly reconnected field lines and the traditional low-altitude identification of the particle signature of the cusp/cleft region made by Newell et al. (1992). However, although the region identified as the 'cusp proper' by Newell and Meng (1992) is almost identical in spatial and temporal extent to the region where the coherent radar signature of newly opened field lines is most likely to be observed, radar signatures of newly opened field lines can be observed over a much larger area than the 'cusp proper' region, just less frequently than at the peak. Also, the study of the duration of the signature of newly reconnected field lines using the entire radar f.o.v, suggests that single-point measurements cannot accurately determine the size of the ionospheric footprint of magnetospheric source regions in a highly variable ionosphere. As the boundary of the 'cusp proper' has been interpreted as representing the extent of the merging gap, this indicates that the size of the active X-line as inferred from the coherent radar signature of newly reconnected field lines can be far greater than the merging gap predicted by the Newell and Meng model. This larger extent of the merging gap is more consistent with the observed poleward convective velocities.

The new model of low-altitude particle signatures as proposed by Lockwood (1997) and Onsager and Lockwood (1997), gives a convincing explanation of the occurrence distribution of the coherent scatter signature of newly reconnected magnetic field lines. It explains why the size of the cusp proper is a partially arbitrary region and predicts that it is smaller than the ionospheric footprint of the merging gap. This new model of dayside precipitation provides a unified theory to explain both the radar observations and the low-altitude satellite observations of the dayside precipitation regimes

\subsection{Seasonal variations}

The normalised occurrence of PIFs observed every month by the radar run in a normal-scan mode is 
illustrated in Fig. 6. There is clearly a sharp decline in the number of PIFs observed during summer months and a peak in observations at the autumn and spring equinoxes. Milan et al. (1997) performed a study of the variation in backscatter occurrence distributions observed by the CUTLASS radar per calendar month, overplotted on Fig. 6 is the backscatter occurrence distribution for the pre-noon sector (at the time and latitude at which the signature of newly-opened flux is most likely to be observed (see Fig 4)). The rate of backscatter occurrence shows a clear peak at the spring equinox, with a minimum in the rate of occurrence in the summer.

The minimum in the rate of backscatter occurrence in the summer months, as reported by Milan et al. (1997), is due to the seasonal variation in the propagation of radio waves in the ionosphere. The soft energy particles associated with open field lines precipitate into the highlatitude F-region ionosphere. During the summer months it is difficult for HF radars to sound the distant F-region, as photoionisation increases the ionospheric ionisation concentration, resulting in the radio waves being more strongly refracted in the ionosphere, thus requiring the propagated rays to make more hops in order to sound the distant F-region ionosphere. Any increase in ionised particles in the $\mathrm{D}$ region ionosphere also results in an increase in the rate of absorption of the radio wave. The signal is thus required to pass through the enhanced D-region three or more times leading to a significant amount of signal absorption. During the summer months, observation of the high-latitude ionosphere is thus not usually possible as any returned signal is likely to be too weak to detect.

Propagation effects explain the minimum in the rate of occurrence of PIFs in the summer, but not the equinoctial maxima. Overplotted an Fig. 6 is the monthly distribution of magnetic storm occurrence between 1963 and 1991 (Taylor et al., 1996). There is a peak in the occurrence distribution at the equinoxes and minima in January and July. The authors propose that this semi-annual variation in the occurrence of storms was due to the semi-annual variation in geomagnetic activity. It has long been established that there is a semiannual variation in geomagnetic activity, with peaks observed near the equinoxes (e.g. McIntosh, 1959; Taylor et al., 1996). McIntosh (1959) proposed that this semi-annual variation was created due to a systematic annual variation in the angle between the Earth's dipole axis and the Sun-Earth line. Russell and McPherron (1973) suggested that the semi-annual variation was created by a semi-annual variation in the effective southward component of the IMF.

A convolution of backscatter occurrence and storm occurrence is overplotted in Fig. 6. This distribution has a very similar shape to the PIFs distribution occurrence with a clear peak at the equinoxes and a minimum during the summer months. Thus we believe that the most likely cause of the seasonal variation in the occurrence rate of PIFs within the CUTLASS Finland radar can be explained by a combination of the annual variation of propagation of radio waves in the iono- sphere and the semi-annual variation of dayside reconnection at the magnetopause. It is noted that more PIFs are observed at the autumn equinox than at the spring equinox. The authors believe that this is probably related to the fact that the IMF $B_{y}$ component was positive for $71 \%$ of the intervals presented in this study (see Provan et al., 1999), but further discussion on this subject is beyond the scope of our current study, and will await improved data coverage. It is possible that the observed seasonal variation of PIFs is related to the creation of polar cap patches of enhanced plasma density during flux transfer events (Lockwood and Carlson, 1992). Such polar cap patches are produced by solar photoionisation on the dayside and would thus exhibit variations with solar luminosity. Valladares et al. (1996) have modelled the formation of the polar cap patches using large plasma flows. However, a study of the relationship between seasonal variation of polar cap patches and PIFs is also beyond the scope of this study.

Figure 7 illustrates the average time of initial observation of poleward moving transients per month as observed by the radar when run in a normal-scan mode. The error bars marked on the graph is equal to the standard deviation at each data point. Initial observations of the graph suggests that on average the initial observations of PIFs occurred earliest in the day during the early summer months (0700 MLT in April) and latest in the day during the winter months (1130-1200 MLT in December/January). A confidence test was performed on the time of initial observation of the transients in the winter months (December, January and February), and in the summer months (April, May and June), to make sure that the time of initial observation of the transient signature was significant. The difference in the time of initial observation of the PIFs is significant at the $99 \%$ confidence level. This again is probably due to seasonal effects on the propagation of radio waves in the ionosphere. In the summer, it appears to be all but impossible to sound the ionosphere in the day-time due to the high-rate of D-region absorption or lack of irregularities due to high Pedersen conductivity and very smooth ionosphere due to insulation. At night the D region completely disappears while the electron density in the $\mathrm{F}$ region decreases slowly. Although the $\mathrm{F}$ region electron density increases rapidly after sunrise, it will be possible for HF radars to observe PIFs occurring in the very early morning. This is just one example of how the radar limits the observation of the ionospheric signature of newly reconnected field lines, potentially underestimating the MLT extent of newly-opened field lines.

\section{Conclusions}

1. This study involves a new method of mapping the dayside ionospheric regions using HF radar data. Pulsed ionospheric flows have been detected by the CUTLASS Finland HF radar, which have been postulated to be the ionospheric signatures of flux transfer events. By utilising the fact that the ionospheric signatures of 
FTEs are observed solely at the footprint of the newly reconnected field lines, these poleward moving transient signatures are used as a means of identifying the regions of the high-latitude ionosphere which map to newly opened field lines.

2. Observing the ionospheric flows with all the radar beams offers a unique possibility to observe the ionospheric footprint of newly-open flux simultaneously for up to $8 \mathrm{~h}$ of local time. The azimuthal extent of the observed pulsed ionospheric flows had a peak at $4-4.5 \mathrm{~h}$ magnetic local time $(\sim 1500 \mathrm{~km})$, while for $28 \%$ of observations the signature extended between 8 and $13 \mathrm{~h}$ MLT (3000-4700 km). The distribution of the temporal duration of pulsed ionospheric flow observed with beam 5 only had a peak at $2.5-3 \mathrm{~h}$. These results would suggest that due to the temporal variability of the region of newly opened flux it is impossible to accurately measure the extent of the ionospheric footprint of newly opened field lines using single point measurements.

3 . The data show a strong seasonal variation in the occurrence rate of PIFs and the time of initial observations of the PIFs, with a minimum in the rate of observation of PIFs in the solstices and maxima at the equinoxes. This annual variation can be attributed to a combination of seasonal effects on the propagation of $\mathrm{HF}$ radio waves in the ionosphere, specifically the increased rate of F-region refraction and D-region absorption during the summer months. The semi-annual variation in geomagnetic activity, as modelled by McIntosh (1959) and Russell and McPherron (1973), results in equinoctial maxima in the rate of geomagnetic activity driven by reconnection, and hence in the observation of ionospheric FTE signatures.

4. A comparison between the statistical pattern of the occurrence of coherent radar signatures of newly opened field lines and the traditional low-altitude satellite identification of particle signatures associated with the cusp, suggests that the extent of the merging gap as predicted by Newell and Meng $(1988,1992)$ is far smaller than the size of the merging gap as inferred from the coherent scatter radar data. These results would suggest that the division of the cusp into different magnetospheric source regions based upon the characteristics of energetic particle precipitation as suggested by Newell and Meng $(1988,1992)$ is not always accurate.

5 . The new model of low-altitude particle signatures as proposed by Lockwood (1997) and Onsager and Lockwood (1997) offers a unified theoretical framework to explain the dayside precipitation regions. This new model predicts that the reconnection merging gap is much greater than the region defined as the cusp proper by Newell and Meng, and this is clearly verified by our observations. This model also demonstrates that the boundary of the cusp proper is to some extent an arbitrary one, determined by the density of the magnetosheath particle density, and is not truly representative of the size of the reconnection region.

Acknowledgements. CUTLASS is supported by PPARC, the Swedish Institute for Space Physics, Uppsala, and the Finnish Meteorological Institute. GP is supported on PPARC grant
GR/L00865. The authors wish to thank Dr. M. Lockwood for useful discussions on modelling plasma precipitation associated with the cusp.

Topical Editor D. Alcaydé thanks A.S. Rodger for his help in evaluating this paper.

\section{References}

Baker, K. B., and S. Wing, A new magnetic coordinate system for conjugate studies at high latitudes, J. Geophys. Res., 94, 91399142, 1989.

Baker, K. B., J. R. Dudeney, R. A. Greenwald, M. Pinnock, P. T. Newell, A. S. Rodger, N. Mattin and C.-I. Merg, HF radar signature of the cusp and low-latitude boundary layer, J. Geophys Res, 100, 7671, 1995.

Cowley, S. W. H., Solar wind control of magnetospheric convection, in Achievement of the International Magnetospheric Study, IMS, p. 483, Noorwijk, Netherlands, 1984.

Cowley, S. W. H., and M. Lockwood, Excitation and decay of solar wind-driven flows in the magnetosphere-ionosphere system, Ann. Geophysicae, 10, 103, 1992.

Cowley, S. W. H., M. P. Freeman, M. Lockwood, and M. F. Smith, The ionospheric signature of flux transfer events, in ClusterDayside Polar Cusp, ESA SP-330, 105, 1991.

Cowley, S. W. H., J. P. Morreli, M. P. Freeman, M. Lockwood, and M. F. Smith, Excitation and decay of flows in the magnetosphere-ionosphere system due to magnetic reconnection at the dayside magnetopause and in the geomagnetic tail, in Proceedings of the International Conference on Substorms, ESA SP-335, p. 117, 1992.

Crooker, N. U., F. T. Toffoletto, and M. S. Gussenhoven, Opening the cusp, J. Geophys. Res., 96, 4397-3503, 1991.

Elphic, R. C., and C. T. Russell, ISEE-1 and -2 observations of the magnetopause, in Magnetospheric Boundary Layers, ESA SP$148,1979$.

Goertz, C. K., E. Nielsen, A. Korth, K. -H. Glassmeier, C. Haldoupis, P. Hoeg, and D. Hayward, Observations of a possible signature of flux transfer events, J. Geophys. Res., 90, 4069-4078, 1985.

Glassmeier, K. H., and M. Stellmacher, Mapping flux transfer events to the ionosphere, Adv. Space Res., 18, 8151-8160, 1996.

Greenwald, R. A., W. Weiss, E. Nielsen, and N. R. Thomson, STARE a new radar auroral backscatter experiment in northern Scandinavia, Radio Sci., 13, 1021-1039, 1978.

Greenwald, R. A., K. B. Baker, J. R. Dudeney, M. Pinnock, T. B. Jones, E. C. Thomas, J.-P. Villain, J.-C. Cerisier, C. Senior, C. Hanuise, R. D. Hunsucker, G. Sofko, J. Koehler, E. Nielsen, R. Pellinen, A. D. M. Walker, N. Sato, and H. Yamagishi, Darn/Superdarn: a global view of the dynamics of high-latitude convection, Space Sci. Rev., 71, 761-796, 1995.

Haerendel, G., G. Paschmann, N. Sckopke, H. Rosenbauer, and P. C. Hedgecock, The frontside boundary layer of the magnetopause and the problem of reconnection, J. Geophys. Res., 83, 3195, 1978.

Heikkila, W. J., and J. D. Winnigham, Penetration of magnetosheath plasma to low altitudes through the dayside magnetospheric cusps, J. Geophys. Res., 76, 883, 1971.

Heppner, J. P., and N. C. Maynard, Empirical high-latitude electric field models, J. Geophys. Res., 92, 4467-4489, 1987.

Lockwood, M., Modelling the high-latitude ionosphere for timevarying plasma convection, Proc. I.E.E (H), 140, 2, 91-100, 1993.

Lockwood, M., Relationship of dayside auroral precipitation to the open-close separatrix and the pattern of convective flow, J. Geophys. Res., 102, 17 475-17 487, 1997.

Lockwood, M., and H. C. Carlson, Production of polar cap electron density patches by transient magnetopause reconnection, Geophys. Res. Lett., 19, 1731-1734, 1992. 
Lockwood, M., and M. N. Wild, On the quasi-periodic nature of magnetopause flux transfer events, J. Geophys Res., 98, 5935$5940,1993$.

Lockwood, M., S. W. H. Cowley, and T. G. Onsager, Ion acceleration at both the interior and exterior Alfvén waves associated with the magnetopause reconnection site: signatures in cusp precipitation, J. Geophys. Res., 101, 21 501-21 513, 1996.

McIntosh, D. H., On the annual variation of magnetic disturbance, Philos. Trans. R. Soc. London, Ser. A., 251, 525-552, 1959.

Milan, S. E., T. K. Yeoman, M. Lester, E. C. Thomas, and T. B. Jones, Initial backscatter occurrence statistics from the CUTLASS HF radars, Ann. Geophysicae, 15, 703-718, 1997.

Moen, J., P. E. Sandholt, M. Lockwood, W. F. Denig, U. P. Lovhaug, B. Lybekk, A. Egeland, D. Opsvik, and E. FriisChristensen, Events of enhanced convection and related dayside auroral activity, J. Geophys. Res., 23, 917-934, 1995.

Newell, P. T., and C.-I. Meng, The cusp and the cleft/boundary layer: low-altitude identification and statistical local time variation, J. Geophys. Res., 93, 14 549-14 556, 1988.

Newell, P. T., and C.-I. Meng, Mapping the dayside ionosphere to the magnetosphere according to particle precipitation characteristics, Geophys. Res. Lett, 19, 609-612, 1992.

Newell, P. T., C. -I. Meng, and D. G. Sibeck, Some low-altitude cusp dependencies of the interplanetary magnetic field, J. Geophys. Res., 94, 8921-8927, 1989.

Neudegg, D. A., T. K. Yeoman, S. W. H. Cowley, G. Provan, G. Haerendel, W. Baumjohann, U. Auster, K.-H. Fornacon, E. Georgescu, C. J. Owen, A flux transfer event observed at the magnetopause by the Equator-S spacecraft and in the ionosphere by the CUTLASS HF radar, (Ann. Geophysicae, in press, 1999).

Onsager, T. G., and M. Lockwood, High-latitude particle precipitation and its relationship to magnetospheric source regions, Space Sci. Rev., 80, 77-107, 1997.

Pinnock, M., A. S. Rodger, J. R. Dudeney, F. Rich, and K. B. Baker, High spatial and temporal resolution observations of the ionospheric cusp, Ann. Geophysicae, 13, 919-925, 1995.

Provan, G., T. K. Yeoman, and S. E. Milan, CUTLASS Finland Radar observations of the ionospheric signatures of flux transfer events and the resulting plasma flows, Ann. Geophysicae, 16, 1411-1412, 1998.

Provan, G., T. K. Yeoman, and S. W. H. Cowley, The influence of the IMF By component on the location of pulsed flows in the dayside ionosphere observed by an HF radar, Geophys. Res. Lett., 26, 521-524, 1999.

Reiff, P. H., R. A. Wolf, T. W. Spiro, Y. Kamide, and J. H. King, Comparison of polar cap potential drops estimated from the solar wind and ground magnetometer data: CDAW-6, J. Geophys. Res., 90, 1318-1324, 1985.

Rijnbeek, R. P., S. W. H. Cowley, D. J. Southwood, and C. T. Russell, A survey of dayside flux transfer events observed by the ISEE-1 and -2 magnetometers, J. Geophys. Res., 89, 786-800, 1984.

Rodger, A. S., and M. Pinnock, The ionospheric response to flux transfer events: the first few minutes, Ann. Geophysicae, 15, 685691, 1997.

Rodger, A. S., S. B. Mende, T. J. Rosenberg, and K. B. Baker, Simultaneous optical and HF radar observations of the ionospheric cusp, Geophys. Res. Lett., 22, 2045-2048, 1995.

Russell, C. T., and R. L. McPherron, Semiannual variation of geomagnetic activity, J. Geophys. Res., 78, 92-108, 1973.

Russell, C. T., and R. C. Elphic, Initial ISEE magnetometer results: magnetopause observations, Space Sci. Rev., 22, 681, 1978.

Russell, C. T., and R. C. Elphic, ISEE observations of flux transfer events at the dayside magnetopause, Geophys. Res. Lett., 6, 3336, 1979.

Russell, C. T., G. Le, and H. Kuo, The occurrence rate of flux transfer events, Adv. Space Res., 18, 8197-8205, 1996.

Sandholt, P. E., M. Lockwood, T. Oguti, S. W. H. Cowley, K. S. C. Freeman, B. Lybekk, A. Egeland, and D. M. Willis, Midday auroral breakup and related energy and momentum transfer from the magnetosheath, J. Geophys. Res., 95, 1039-1060, 1990.

Sandholt, P. E., J. Moen, and D. Opsvik, Periodic auroral events at the midday polar cap boundary: implications or solar windmagnetosphere coupling, Geophys. Res. Lett., 19, 1223-1226, 1992.

Siscoe, G. L., and T. S. Huang, Polar cap inflation and deflation, J. Geophys Res., 90, 543-547, 1985.

Taylor, J. R., M. Lester, and T. K. Yeoman, Seasonal variations in the occurrence of geomagnetic storms, Ann. Geophysicae, 14, 286-289, 1996.

Van Eyken, A. P., H. Risbeth, and M. A. Saunders, Initial observations of plasma convection at invariant latitudes of 70-77, J. Atmos. Terr. Phys., 46, 635-641, 1984.

Valladares, C. E., D. T. Decker, R. Sheehan, and D. N. Anderson, Modelling the formation of polar cap patches using large plasma flow, Radio Sci., 31, 573-593, 1996.

Vasyliunas, V. M., Interaction between the magnetospheric boundary layers and the magnetosphere, in Magnetospheric Boundary Layers, Ed. B. Battrick, Eur. Space Agency Spec. Publ., ESA SP-148, 387-393, 1979

Yeoman, T. K., M. Lester, S. W. H. Cowley, S. E. Milan, J. Moen, and P. E. Sandholt, Simultaneous observations of the cusp in optical, DMSP and HF radar data, Geophys. Res. Lett., 24, 2251-2254, 1997. 